\section{Fruit Abscission in Rabbiteye Blueberry in Response to Organ Removal and Mechanical Wounding}

\author{
Tripti Vashisth ${ }^{1}$ and Anish Malladi ${ }^{2}$ \\ Department of Horticulture, University of Georgia, 1111 Miller Plant \\ Sciences, Athens, GA 30602
}

\begin{abstract}
Additional index words. abscission agents, fruit detachment, harvest aid, jasmonates, plant growth regulator
\end{abstract}

\begin{abstract}
Fruit abscission in blueberry (Vaccinium sp.) occurs at the pedicel/peduncle junction (PPJ). Growth regulators such as methyl jasmonate (MeJa) and ethephon accelerate the progression of abscission at this zone. It is not known whether the abscission zone at the PPJ is sufficient to perceive and respond to these growth regulator applications or if the fruit and leaf tissues are required to elicit these responses. Furthermore, the effects of injury to the fruit and leaves on fruit detachment responses have not been previously reported in blueberry. In this study, the requirement of the fruit and leaves to respond to MeJa and ethephon applications was investigated through organ removal treatments in rabbiteye blueberry. Removal of the fruit or the fruit and leaves on the branch followed by MeJa application delayed the progression of abscission at the PPJ suggesting that the fruit tissue is required only to accelerate the progression of fruit detachment in response to MeJa. Interestingly, the extent of fruit/pedicel detachment in response to ethephon applications was higher in the organ removal treatments compared with the control indicating that the PPJ was sufficient to perceive and respond to ethephon and that wounding caused by organ removal synergistically enhanced fruit abscission in response to ethephon. Mechanical wounding of the fruit by removing the distal half of the berry resulted in accelerated fruit detachment at the PPJ. Detachment of non-injured fruit was unaffected by mechanical wounding of adjacent fruit. These data suggest that wounding generates a local signal capable of accelerating fruit abscission at the PPJ. This information may have implications for fruit retention or drop in response to injury to the fruit as caused by herbivore feeding or as a result of insects and pathogens.
\end{abstract}

Abscission is a physiological process involving the detachment of an organ from the plant at specific regions termed abscission zones (AZs; Roberts et al., 2002). Organ detachment through abscission may be the result of an intricately controlled developmental process to achieve its removal on completion of its function or to achieve the dispersal of its contents. Alternatively, abscission may occur in response to different types of stress such as drought or wounding resulting from insect, pathogen, or herbivore attack (Taylor and Whitelaw, 2001). Multiple phytohormones such as auxins, ethylene, jasmonates, and abscisic acid regulate the signaling mechanisms leading to the activation and progression of abscission (Hartmond et al., 2000; Kim et al., 2013; Roberts et al., 2002; Taylor and Whitelaw, 2001). External application of some of these growth regulators

Received for publication 12 Aug. 2014. Accepted for publication 17 Sept. 2014.

This research was supported by the U.S. Department of Agriculture-Specialty Crops Research Initiative Grant: 2008-51180-19579.

${ }^{1}$ Current address: Citrus Research and Education Center, University of Florida, Lake Alfred, FL 33850 .

${ }^{2}$ To whom reprint requests should be addressed; e-mailmalladi@uga.edu. affects the progression of abscission. Such applications can be used to control the detachment of organs such as the fruit and are potentially useful as harvest aids (Burns et al., 2005; Fidelibus et al., 2007; Malladi et al., 2012).

Blueberry has a production value of over $\$ 750$ million in the United States and has emerged as a major fruit crop in the southeastern United States over the last decade (U.S. Department of Agriculture, 2013). Recent studies have started exploring the regulation of fruit detachment in blueberry with the aim of developing abscission agents that can be used to improve mechanical harvesting efficiency (Malladi et al., 2012, 2013; Vashisth and Malladi, 2013). Developmentally regulated mature fruit detachment through the physiological process of abscission occurs within the AZ located at the PPJ (Vashisth and Malladi, 2013). Application of MeJa (20 mM), an oxylipin, and ethephon (1000 mg. $\mathrm{L}^{-1}$ ), an ethylene-releasing compound, results in rapid (24 to $48 \mathrm{~h}$ ) and extensive abscission of mature blueberry fruit (Malladi et al., 2012). Fruit detachment as a result of the application of these growth regulators occurs at the PPJ indicating that these compounds accelerate the progression of abscission (Malladi et al., 2012; Vashisth and Malladi, 2013). However, it is unclear if the PPJ perceives and responds to these growth regulator applications or whether the fruit and leaf tissues are required for such responses. It is possible that these growth regulator applications are directly perceived at the AZ within the PPJ thereby facilitating an abscission response. Alternatively, the perception of these growth regulators may occur within the berry or within leaves on the branch leading to the generation of a signal that is translocated to the $\mathrm{AZ}$ at the PPJ, thereby activating abscission.

Abscission may also be induced or accelerated as a result of injury as a result of pathogen, insect, or herbivore attack or as a result of mechanical wounding (Taylor and Whitelaw, 2001). In citrus (Citrus sinensis), mechanical wounding of the flavedo of the mature fruit resulted in a decrease in the fruit detachment force and an increase in the extent of fruit drop (Kostenyuk and Burns, 2004). Injury to the fruit may result in the generation and release of a variety of signals including phytohormones such as ethylene and jasmonates (Howe, 2004). Some of these signals may translocate over long distances and induce responses within non-injured organs (Howe, 2004; Schilmiller and Howe, 2005). Potentially, such signals may activate or accelerate the abscission response. In blueberry, significant injury to the fruit is often observed as a result of bird feeding. It is unknown whether such injury results in the abscission of the affected fruit and whether it generates signals that cause adjacent fruit to respond similarly.

The objectives of this study were: 1) to determine whether the AZ at the PPJ in the mature fruit is by itself sufficient to perceive and respond to abscission agent applications; and 2) to study the effect of mechanical injury to mature fruit on the abscission of the affected fruit and adjacent non-injured fruit. Organ removal treatments followed by growth regulator applications and mechanical wounding of mature fruit and leaves in rabbiteye blueberry (Vaccinium ashei Reade) were used to address these objectives.

\section{Materials and Methods}

Expt. 1. Rabbiteye blueberry plants of the cultivar, Climax, planted in 2008 at the University of Georgia Horticulture Farm in Watkinsville, GA, were used in this study in 2013. The experiment was performed when the majority of the fruit on the plant was mature (blue/black). A split-plot experimental design with four replicates was used. The whole-plot factor was completely randomized and consisted of the following growth regulator applications: 1) control (water); 2. MeJa (20 mм; Sigma-Aldrich, St. Louis, MO); and 3) ethephon (1000 mg. $\mathrm{L}^{-1}$; Bayer CropScience, Kansas City, MO). All applications were made along with $0.15 \%$ of Latron B-1956 (Rohm and Haas, Philadelphia, PA) as the adjuvant. All spray applications were performed $\approx 0900 \mathrm{~h}$ until runoff using a hand sprayer. The average daily temperature on the day the spray applications 
were performed was $25.6{ }^{\circ} \mathrm{C}$ and ranged from 23.2 to $27.8^{\circ} \mathrm{C}$ during the duration of the experiment. On each plant, four branches, each consisting of $\approx 25$ to 45 fruit, were tagged and randomly assigned to one of four organ removal treatments (split-plot factor). On all these branches, immature fruit (green and pink) were removed $\approx 1 \mathrm{~h}$ before the application of treatments. The organ removal treatments consisted of: 1) no organ removal (NR); 2) fruit removal (FR); 3) leaf removal (LR); and 4) fruit and leaf removal (FLR). The FR treatment consisted of hand detachment of all mature fruit on the branch at the junction of the pedicel and the berry resulting in the retention of only the pedicels on the branch. The LR treatment involved the removal by hand of all leaves present on the fruiting portion of the branch and up to $\approx 15 \mathrm{~cm}$ from it. The FLR treatment involved the removal of both the fruit and the leaves on the branches as described previously for the FR and LR treatments. The organ removal treatments were applied $\approx 30 \mathrm{~min}$ before the growth regulator treatments. The number of fruit or pedicels on the tagged branches was counted to determine the extent of fruit/pedicel drop in response to the growth regulator and organ removal treatments. A preliminary experiment performed in 2012 using 'Powderblue' rabbiteye blueberry plants indicated that pedicels in treatments involving fruit removal often remained loosely attached to the branch although abscission had progressed, potentially as a result of the absence of the weight of the fruit to complete the detachment process. Hence in the 2013 experiment with 'Climax', the pedicels were brushed lightly before the fruit/pedicel count in all the treatments to promote complete detachment of abscised organs.

Expt. 2. The effect of organ injury on fruit detachment characteristics was determined in the rabbiteye cultivars, Premier and Powderblue. Blueberry bushes planted in 2010 at the University of Georgia Horticulture Farm in Watkinsville, GA, were used in this study in 2013. In the study with 'Premier', four treatments were applied when the majority of the fruit on the plant was mature. Four single-plant replicates were used in this study in a completely randomized design. On each plant, one branch with 25 to 50 fruit was selected and subjected to the organ injury treatment. Only mature fruit were used in this experiment. Approximately $1 \mathrm{~h}$ before the treatment, all immature fruit were removed from the selected branch. The treatments were: 1) control (no organ injury); 2) fruit injury; 3) 50\% fruit injury; and 4) leaf injury. For the fruit injury treatment, all fruit on the branch were cut in half using a razor blade such that the top half of the fruit was left attached to the branch through the pedicel and the bottom half was discarded. For the $50 \%$ fruit injury treatment, $\approx 50 \%$ of randomly selected fruit on the branch was injured as described previously, whereas the remaining fruit were not injured. For the leaf injury treatment, the distal half of the leaf blades on all of the leaves on the branch was cut and removed using scissors. The fruit were not injured in this treatment. The number of fruit on the branch was counted at the beginning of the experiment and until 2 weeks after treatment. The average daily temperature during the duration of the experiment ranged from 23.2 to $27.8^{\circ} \mathrm{C}$. The point of detachment of the fruit was noted. This experiment was repeated in the rabbiteye blueberry cultivar, Powderblue. In this study all the treatments were performed as described previously except for the leaf injury treatment. This treatment was not performed because it did not appear to alter the fruit detachment responses in the study with 'Premier'. Four replicates in a completely randomized design were used in this study. In this experiment, fruit drop was monitored until $10 \mathrm{~d}$ after treatment. The average daily temperature during the duration of the experiment ranged from 23.4 to $27.3{ }^{\circ} \mathrm{C}$.

Statistical analyses. Statistical analyses were performed using SAS (Version 9.3; Cary, NC) and SigmaPlot 11 (San Jose, CA). Percent fruit drop data were transformed using arcsine square-root transformation before data analyses. Data from Expt. 1 were analyzed using PROC MIXED in SAS. The interaction effects between the growth regulator treatment and the organ removal treatment were significant at all stages after treatment. The simple effects of the organ removal treatments within each growth regulator treatment were tested using the test of effect slices $(\alpha=0.05)$. When this test was significant, pairwise comparisons were used to separate the means of the organ removal treatments. Data from Expt. 2 were analyzed using analysis of variance. Comparison of means within each time point after treatment was performed using Fisher's least significant difference $(\alpha=0.05)$.

\section{Results}

Expt. 1. In 'Climax', fruit drop within the control treatment reached up to $37 \%$ by $192 \mathrm{~h}$ after treatment (Fig. 1, NR treatment). None of the organ removal treatments affected the extent of fruit drop in the control treatment.

Application of MeJa resulted in rapid and extensive fruit drop in 'Climax' (Fig. 1). The extent of fruit drop in response to MeJa application was up to $64 \%$ by $24 \mathrm{~h}$ after treatment and reached up to $91 \%$ by $192 \mathrm{~h}$ after treatment (NR). Removal of the fruit at the pedicel-berry junction (FR treatment) followed by MeJa application resulted in an altered detachment response. In the FR treatment, 2- to 3-fold lower detachment of the pedicels was observed between 24 and $96 \mathrm{~h}$ after treatment in comparison with the NR treatment. At later stages, pedicel detachment in the FR treatment was not different from that in the NR treatment. Leaf removal in the LR treatment resulted in fruit drop similar to that observed in the NR treatment and was significantly different from that in the other treatments until $96 \mathrm{~h}$ after treatment. Removal of the fruit and the leaves in the FLR treatment resulted in a detachment response largely similar to that observed in the FR treatment. The FLR treatment resulted in significantly lower pedicel detachment than the fruit detachment in the NR treatment by almost 5- to 2-fold at 24 and $48 \mathrm{~h}$ after treatment, respectively. However, from $72 \mathrm{~h}$ after treatment, the extent of pedicel/fruit detachment was not different between the FLR and the NR treatments.

Application of the growth regulator ethephon resulted in extensive fruit drop in 'Climax' (Fig. 1). In the NR treatment, ethephon application resulted in $65 \%$ fruit drop by $192 \mathrm{~h}$ after treatment. Interestingly, the organ removal treatments, FR, LR, and FLR, resulted in higher pedicel/fruit drop than that in the NR treatment in response to ethephon. Between 24 and $96 \mathrm{~h}$ after treatment, the extent of pedicel/fruit drop in these organ removal treatments was greater than 2 -fold that in the NR treatment. The organ removal treatments resulted in up to $100 \%$ detachment in response to the ethephon treatment by $96 \mathrm{~h}$ after application.

Expt. 2. The effect of mechanical wounding of fruit or leaves on fruit drop was studied in 'Premier' (Fig. 2). In the control, less than $20 \%$ fruit drop was observed within the first $7 \mathrm{~d}$ of observation and $52 \%$ of the fruit dropped by $14 \mathrm{~d}$ after treatment. In the treatment in which all the fruit on the branch was injured, a clear increase in the rate of fruit drop was evident. By $3 \mathrm{~d}$ after treatment, $40 \%$ of the fruit in this treatment detached and $88 \%$ of the fruit detached by $7 \mathrm{~d}$ after treatment. Almost all of the wounded fruit $(99 \%)$ detached by the end of the experiment ( $14 \mathrm{~d})$. Injuring $50 \%$ of the fruit on the branch resulted in significantly higher fruit drop (greater than 2.5-fold) than that in the control from $7 \mathrm{~d}$ after treatment. The extent of fruit drop in the non-injured fruit within this treatment was not different from that in the control treatment (data not shown). The leaf injury treatment did not alter the extent of fruit drop in comparison with the control treatment.

In 'Powderblue', the control fruit displayed up to $36 \%$ fruit drop by $10 \mathrm{~d}$ after treatment (Fig. 3). In the fruit injury treatment in which all fruit on the branch was cut in half, the extent of fruit drop was greatly accelerated, similar to that observed in the 'Premier' study. Greater than $55 \%$ of these fruit detached at $2 \mathrm{~d}$ after treatment and the extent of fruit drop was greater than $96 \%$ by $5 \mathrm{~d}$ after treatment. In the fruit injury treatment in which only half the fruit on the branch were injured, fruit detachment was significantly higher (greater than 3-fold) than that in the control from $5 \mathrm{~d}$ after treatment. The non-injured fruit on the branch within this treatment displayed a fruit drop response similar to that of the control (data not shown). Fruit detachment in response to mechanical wounding of the fruit occurred primarily at the PPJ.

\section{Discussion}

In the organ removal experiment, $37 \%$ fruit abscission occurred in the control treatment by $8 \mathrm{~d}$ after treatment indicating the 


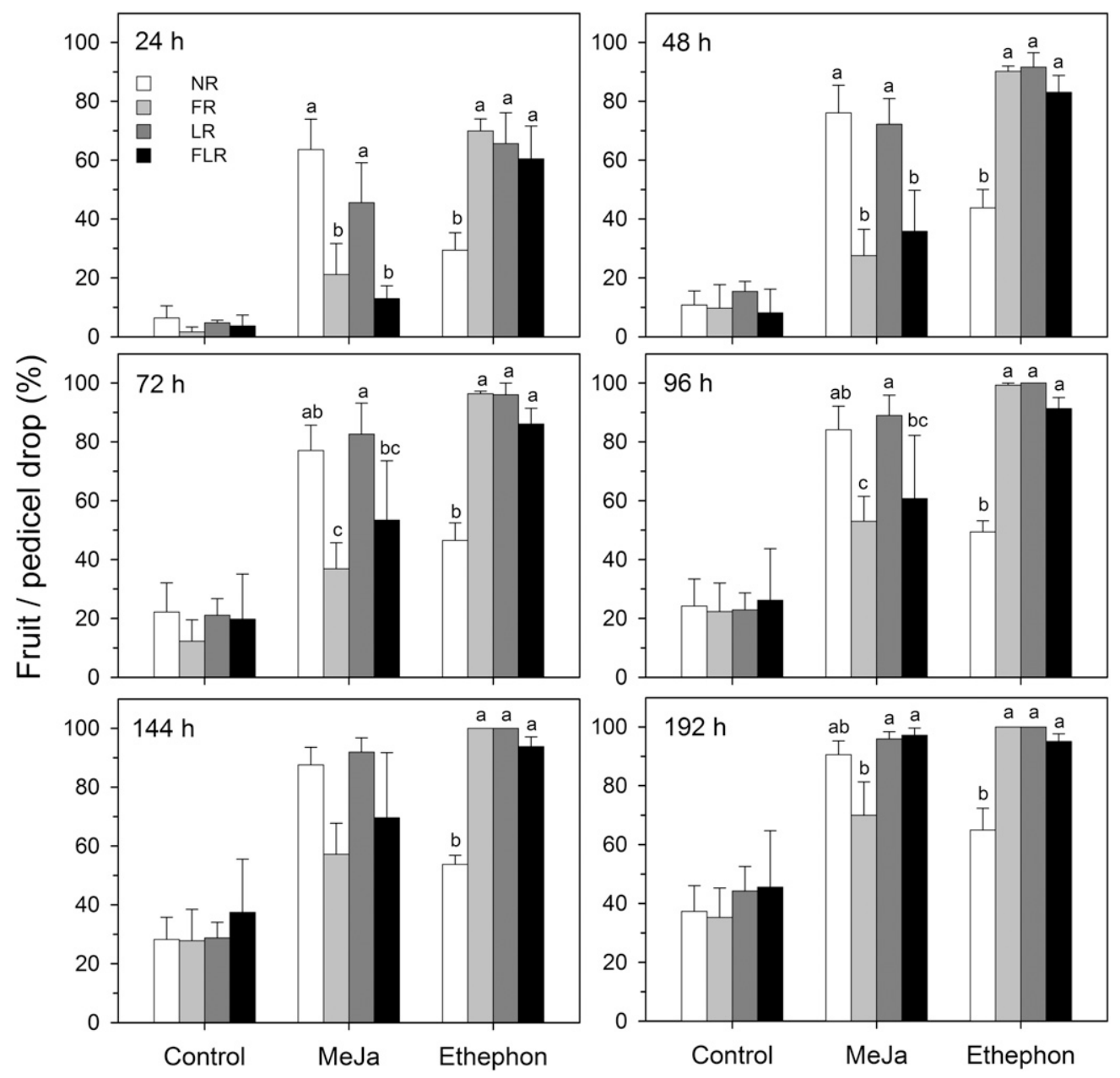

Fig. 1. Fruit or pedicel detachment at the pedicel/peduncle junction (PPJ) in response to abscission agent applications and organ removal treatments in 'Climax'. Four organ removal treatments were performed: $\mathrm{NR}=$ no removal; $\mathrm{FR}=$ fruit removal at the pedicel/berry junction; $\mathrm{LR}=$ leaf removal on the branch with fruit; $\mathrm{FLR}=$ fruit and leaf removal (as described previously). Methyl jasmonate (MeJa; $20 \mathrm{~mm}$ ) and ethephon (1000 $\mathrm{mg} \cdot \mathrm{L}^{-1}$ ) along with the adjuvant were applied after the organ removal treatments. The control was sprayed with water and adjuvant only. Fruit or pedicels retained on the treated branches were counted and these data were used to determine the percentage fruit/pedicel drop. Error bars indicate the sEMs $(n=4)$. Similar letters above the bars indicate that the means of the organ removal treatments within a growth regulator treatment were not significantly different $(\alpha=0.05)$.

extent of natural fruit detachment during this period. None of the organ removal treatments altered the extent of abscission at the PPJ. These data indicate that the extent of injury caused by the removal of the fruit, leaves, or both was not sufficient to accelerate the fruit abscission response in the mature fruit.

Application of the abscission agent MeJa resulted in accelerated fruit detachment. Greater than $60 \%$ of the mature fruit abscised within $1 \mathrm{~d}$ after treatment with MeJa. These data are consistent with previous studies in which rapid detachment of mature fruit in response to MeJa application was reported in blueberry (Malladi et al., 2012; Vashisth and Malladi, 2013). The PPJ has previously been demonstrated to be the point of fruit detachment through the physiological process of abscission in blueberry (Vashisth and Malladi, 2013). Removal of the fruit at the fruit/pedicel junction (FPJ) in the FR and FLR treatments resulted in at least $70 \%$ abscission at the PPJ indicating that the abscission zone at the PPJ was by itself competent to respond to the MeJa applications and initiate detachment. These data clearly indicate that in mature fruit, the abscission zone at the PPJ is developmentally mature and able to respond to signals that activate or promote abscission. However, presence of the mature fruit tissue was clearly required to accelerate the abscission response at the PPJ. Removal of the fruit in the FR treatment especially resulted in lower detachment at the PPJ than that in the NR treatment until at least $4 \mathrm{~d}$ after treatment. Presence of the mature fruit may allow for a greater surface area for the absorption of MeJa, thereby resulting in an accelerated abscission response. Alternatively, presence of the mature fruit may allow for a jasmonate-dependent signal generated within the fruit (in response to MeJa) to translocate to the PPJ and add to similar signals generated within this location, thereby accelerating the progression of abscission.

Ethephon application resulted in mature fruit detachment by up to $65 \%$ within $8 \mathrm{~d}$ after treatment. These data are consistent with previous reports on the effects of ethephon on blueberry fruit detachment (Malladi et al., 2012; Vashisth and Malladi, 2013). Interestingly, removal of the fruit, leaves, or both resulted in enhanced abscission at the PPJ by up to 2-fold, clearly indicating that the abscission zone at the PPJ is sufficient to respond to ethephon and initiate abscission. Organ removal may lead to the generation of ethylene and/or jasmonate-dependent signals. Such wound-induced generation of ethylene and jasmonate signals has been previously reported in other plant systems such as Arabidopsis and tomato ( $\mathrm{Li}$ et al., 2002; O'Donnell et al., 1996). Wound signals generated through organ removal treatments may act synergistically with external ethephon applications resulting in higher abscission within these treatments. These signals alone were, however, not sufficient to enhance the abscission response because the organ removal treatments did not differ in the extent of abscission in the control treatment.

Mechanical wounding of the fruit through the removal of the bottom half of the fruit resulted in accelerated abscission of the injured fruit in 'Premier' and 'Powderblue'. Up to $90 \%$ of the wounded fruit detached within $5 \mathrm{~d}$ after injury indicating a rapid abscission 


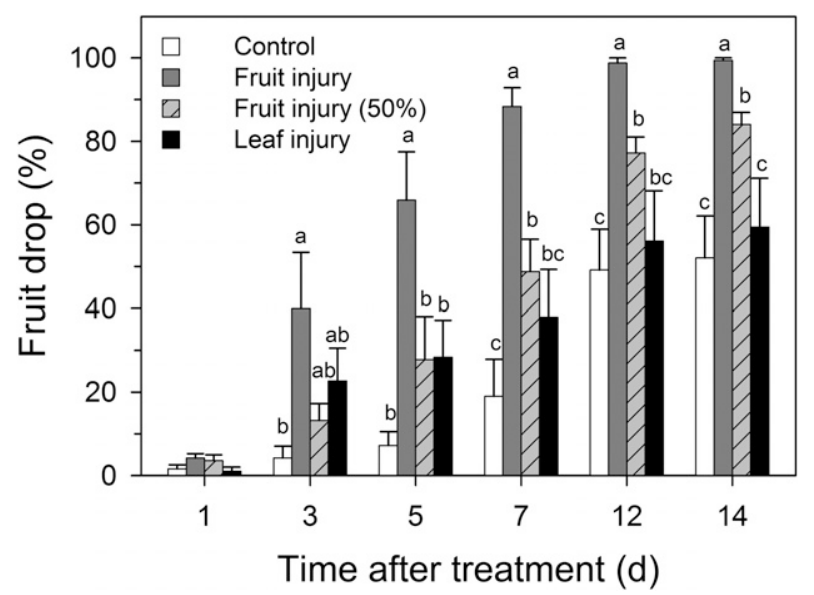

Fig. 2. Fruit abscission in response to mechanical wounding in 'Premier'. Four treatments were performed. Control $=$ no injury; Fruit injury $=$ all mature fruit were mechanically wounded by removal of the distal half of the berry; Fruit injury $(50 \%)=$ half of the fruit on the branch were randomly subjected to the mechanical wounding treatment as described previously; and Leaf injury $=$ all leaves on the branch with the fruit were mechanically wounded. The error bars indicate the SEMs $(n=4)$. Similar letters above the bars indicate that the means within the given time after treatment were not significantly different using Fisher's least significant difference $(\alpha=0.05)$.

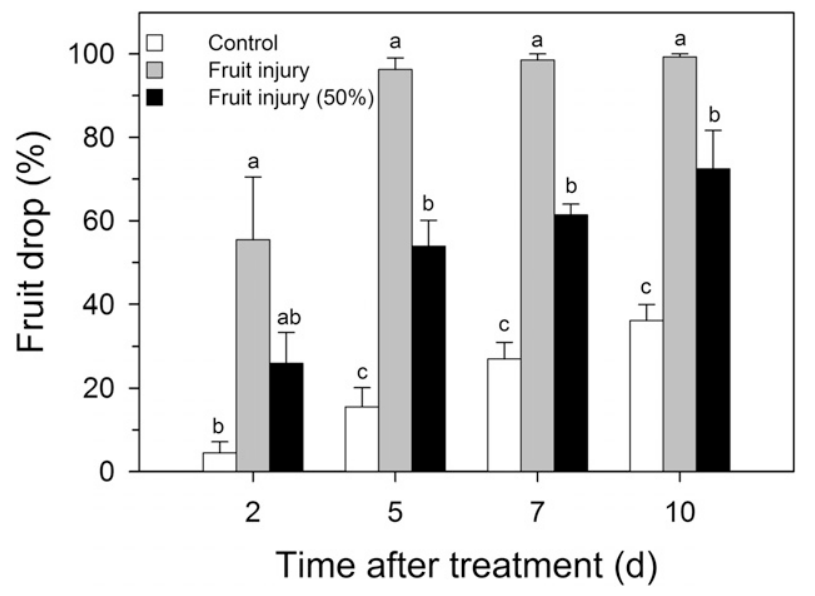

Fig. 3. Fruit abscission in response to mechanical wounding in 'Powderblue'. Three treatments were performed on mature fruit. Control $=$ no injury; Fruit injury $=$ all mature fruit were mechanically wounded by removing the distal half of the berry; Fruit injury $(50 \%)=$ half of the fruit on the branch were mechanically wounded as described previously. The error bars indicate the SEMS $(n=4)$. Similar letters over the bars indicate that the means within the given time after treatment were not significantly different as determined by Fisher's least significant difference $(\alpha=0.05)$.

response as a result of mechanical wounding. The majority of the fruit detachment occurred at the abscission zone within the PPJ. These data suggest the generation of a wound-induced signal that accelerates the progression of abscission at the PPJ. Injury to the fruit may trigger the generation of a signal, which is rapidly translocated to the PPJ where it may interact with native signals to promote the progression of abscission. Similarly, mechanical wounding of the fruit flavedo has been shown to reduce the fruit detachment force and to accelerate mature fruit abscission in citrus (Kostenyuk and Burns, 2004). Interestingly in the current study, fruit abscission as a result of mechanical wounding to the fruit was restricted to the injured fruit. Non-wounded fruit adjacent to the injured fruit on the same branch did not display an increase in the rate of fruit detachment. These data suggest that the potential signal generated as a result of mechanical wounding is active only over a local region and is not communicated over a long distance. In citrus, the fruit detachment effect of mechanical wounding of the fruit flavedo was dependent on the proximity of the injury to the abscission zone, suggesting that the signal was translocated only over a short distance (Kostenyuk and Burns, 2004). Additionally, in the current study, mechanical injury to the leaves on the branch did not accelerate the extent of mature fruit detachment. Mechanical wounding of the leaves may not have generated sufficient levels of the abscission signal. Alternatively, the signal may not have translocated over a long distance.
Fruit injury may result in the generation of signals such as ethylene and/or jasmonates, which could potentially accelerate the progression of abscission at the PPJ. Ethylene release is often associated with wounding (Kato et al., 2000; O'Donnell et al., 1996; Ryan and Moura, 2002). Ethylene may, either directly or through interaction with other wound-induced signals, trigger downstream signaling mechanisms resulting in a plant response to wounding (Howe, 2004; O’Donnell et al., 1996), including abscission. Abscission of the injured organ may be a mechanism to prevent the establishment and spread of potential infections at the point of injury (Taylor and Whitelaw, 2001). Ethylene generation as a result of mechanical injury to the fruit has been previously implicated in accelerated mature fruit abscission in citrus where blocking ethylene biosynthesis decreased fruit drop in response to injury (Kostenyuk and Burns, 2004). Additionally, jasmonates are key components of wound signaling in plants (Howe, 2004; Li et al., 2002; Schilmiller and Howe, 2005). Jasmonates act as signals generated at or close to the point of injury and are systemically transported over long distances to induce responses to wounding (Howe, 2004; Schilmiller and Howe, 2005). Jasmonates are involved in regulating fruit detachment responses in various plants such as Arabidopsis (Arabidopsis thaliana), blueberry, and citrus (Hartmond et al., 2000; Kim et al., 2013; Malladi et al., 2012; Vashisth and Malladi, 2013). It is likely that the induction of jasmonate biosynthesis and signaling as a result of fruit injury may accelerate the progression of abscission at the PPJ of the injured fruit. Quantification of ethylene and jasmonates is essential to determine if these hormones are involved in mediating the mechanical wounding-related fruit abscission responses in blueberry.

Together, the studies described here provide insights into the signaling mechanisms involved in the progression of mature fruit abscission in blueberry. The abscission zone at the PPJ in mature fruit appears to be at a physiological stage sufficient to functionally respond to the application of abscission agents such as MeJa and ethephon, although presence of the mature fruit tissue accelerates such a response in the case of MeJa. Injury to the fruit may generate a local signal that accelerates abscission at the PPJ in the injured mature fruit. This information has potential implications with respect to fruit injury caused by bird feeding. The results from this study suggest that only the injured fruit may abscise in response to such feeding, whereas the adjacent fruit remain largely unaffected.

\section{Literature Cited}

Burns, J.K., R.S. Buker, and F.M. Roka. 2005. Mechanical harvesting capacity in sweet orange is increased with an abscission agent. HortTechnology 15:758-765.

Fidelibus, M.W., K.A. Cathline, and J.K. Burns. 2007. Potential abscission agents for raisin 
table and wine grapes. HortScience 42:16261630.

Hartmond, U., R.C. Yuan, J.K. Burns, A. Grant, and W.J. Kender. 2000. Citrus fruit abscission induced by methyl-jasmonate. J. Amer. Soc. Hort. Sci. 125:547-552.

Howe, G.A. 2004. Jasmonates as signals in the wound response. J. Plant Growth Regul. 23: 223-237.

Kato, M., Y. Hayakawa, H. Hyodo, Y. Ikoma, and M. Yano. 2000. Wound-induced ethylene synthesis and expression and formation of 1-aminocyclopropane-1-carboxylate (ACC) synthase, ACC oxidase, phenylalanine ammonialyase, and peroxidase in wounded mesocarp tissue of Cucurbita maxima. Plant Cell Physiol. 41:440-447.

Kim, J., B. Dotson, C. Rey, J. Lindsey, A.B. Bleecker, B.M. Binder, and S.A. Patterson. 2013. New clothes for the jasmonic acid receptor COI1: Delayed abscission, meristem arrest and apical dominance. PLoS One 8:E60505.

Kostenyuk, I. and J.K. Burns. 2004. Mechanical wounding and abscission in citrus. Physiol. Plant. 122:354-361.

Li, L., C. Li, G.I. Lee, and G.A. Howe. 2002. Distinct roles for jasmonate synthesis and action in the systemic wound response of tomato. Proc. Natl. Acad. Sci. USA 99:64166421.

Malladi, A., T. Vashisth, and L.K. Johnson. 2012. Ethephon and methyl jasmonate affect fruit detachment in rabbiteye and southern highbush blueberry. HortScience 47:1745-1749.

Malladi, A., T. Vashisth, and S. NeSmith. 2013. Development and evaluation of a portable, hand-held mechanical shaker to study fruit detachment in blueberry. HortScience 48: 394-397.

O’Donnell, P.J., C. Calvert, R. Atzorn, C. Wasternack, H.M.O. Leyser, and D.J. Bowles. 1996. Ethylene as a signal mediating the wound response of tomato plants. Science 274:1914-1917.

Roberts, J.A., K.A. Elliott, and Z.H. GonzalezCarranza. 2002. Abscission, dehiscence and other cell separation processes. Annu. Rev. Plant Biol. 53:131-158.

Ryan, C.A. and D.S. Moura. 2002. Systemic wound signaling in plants: A new perception. Proc. Natl. Acad. Sci. USA 99:6519-6520.

Schilmiller, A.L. and G.A. Howe. 2005. Systemic signaling in the wound response. Curr. Opin. Plant Biol. 8:369-377.

Taylor, J.E. and C.A. Whitelaw. 2001. Signals in abscission. New Phytol. 151:323-339.

U.S. Department of Agriculture. 2013. Non-citrus fruits and nuts 2012 summary. National Agricultural Statistics Service, Washington, DC.

Vashisth, T. and A. Malladi. 2013. Fruit detachment in rabbiteye blueberry: Abscission and physical separation. J. Amer. Soc. Hort. Sci. 138:95-101. 